\title{
JOHN VON NEUMANN'S WORK IN THE THEORY OF GAMES AND MATHEMATICAL ECONOMICS
}

\author{
H. W. KUHN AND A. W. TUCKER
}

Of the many areas of mathematics shaped by his genius, none shows more clearly the influence of John von Neumann than the Theory of Games. This modern approach to problems of competition and cooperation was given a broad foundation in his superlative paper of 1928 [A]. ${ }^{1}$ In scope and youthful vigor this work can be compared only to his papers of the same period on the axioms of set theory and the mathematical foundations of quantum mechanics. A decade later, when the Austrian economist Oskar Morgenstern came to Princeton, von Neumann's interest in the theory was reawakened. The result of their active and intensive collaboration during the early years of World War II was the treatise Theory of games and economic behavior [D], in which the basic structure of the 1928 paper is elaborated and extended. Together, the paper and treatise contain a remarkably complete outline of the subject as we know it today, and every writer in the field draws in some measure upon concepts which were there united into a coherent theory.

The crucial innovation of von Neumann, which was to be both the keystone of his Theory of Games and the central theme of his later research in the area, was the assertion and proof of the Minimax Theorem. Ideas of pure and randomized strategies had been introduced earlier, especially by Emile Borel [3]. However, these efforts were restricted either to individual examples or, at best, to zero-sum two-person games with skew-symmetric payoff matrices. To paraphrase his own opinion expressed in $[\mathbf{J}]$, von Neumann did not view the mere desire to mathematize strategic concepts and the straight formal definition of a pure strategy as the main agenda of an "initiator" in the field, but felt that there was nothing worth publishing until the Minimax Theorem was proved.

As the leitmotiv of this article, the Minimax Theorem requires at least informal statement at the outset. (A later section will present

Received by the editors February 21, 1958.

1 There are two bibliographies at the end of this article, one of von Neumann's work in the field and the second for other references. Letters in square brackets refer to the von Neumann bibliography and numbers in square brackets refer to the other bibliography. 
its mathematical structure in greater detail.) For any finite zero-sum two-person game in a normalized form, it asserts the existence of a unique numerical value, representing a gain for one player and a loss for the other, such that each can achieve at least this favorable an expectation from his own point of view by using a randomized (or mixed) strategy of his own choosing. Such strategies for the two players are termed optimal strategies and the unique numerical value, the minimax value of the game. This is the starting point of the von Neumann-Morgenstern solution for cooperative games, where all possible partitions of the players into two coalitions are considered and the reasonable aspirations of the opposing coalitions in each partition measured by the minimax value of the strictly competitive twoparty struggle between them. In the area of extensive games, the solution of games with perfect information by means of pure strategies assumes importance only by contrast to the necessity of randomizing in the general case. The Minimax Theorem reappears in a new guise, when von Neumann turned to analyze a linear model of production. Finally, in the hands of von Neumann, it was the source of a broad spectrum of technical results, ranging from his extensions of the Brouwer fixed point theorem, developed for its proof, to new and unexpected methods for combinatorial problems.

In the attempt to encompass von Neumann's broad, yet detailed, contributions to game theory and mathematical economics, this article is divided into two main parts. The first deals with conceptual innovations that are rather strictly bound to the context of game theory and linear economic theory. The second deals in stricter detail with more technical results and computational methods centered on the Minimax Theorem, but of interest in themselves, and also with various mathematical ramifications.

\section{Part I. Conceptual Innovations}

Cooperative games. Von Neumann's first attack on games that offer opportunities for cooperation was made in his 1928 paper [A]. Attempting a parallel to the minimax value for zero-sum two-person games, he sought appropriate numerical values for the players of a zero-sum three-person game. With coalitions possible, strictly competitive conditions no longer prevail, and so he proposed a solution in terms of basic values for the players together with premiums and penalties which depend on which coalition actually forms.

To describe this solution concept, he introduced the idea of a characteristic function, defined on each set $S$ of players, and taking as a value $v(S)$, the minimax value that $S$ is assured in a zero-sum 
two-person game against the complementary set of players. Then the basic values for the three players in a zero-sum three-person game are

$$
\begin{aligned}
& v_{1}=\frac{1}{3}(v(\{1,2\})+v(\{1,3\})-2 v(\{2,3\})), \\
& v_{2}=\frac{1}{3}(v(\{1,2\})+v(\{2,3\})-2 v(\{1,3\})), \\
& v_{3}=\frac{1}{3}(v(\{1,3\})+v(\{2,3\})-2 v(\{1,2\})) .
\end{aligned}
$$

If two players form a coalition against the third, they are each entitled to a premium of $D / 6$, while the third player suffers a penalty of $D / 3$, where

$$
D=v(\{1,2\})+v(\{1,3\})+v(\{2,3\}) .
$$

No further attempt was made in the 1928 paper to solve the general cooperative game with more than three players. However, it was conjectured that the characteristic function provides sufficient data for such a solution. The basic values proposed for $n=3$ find their generalization in the Shapley value [15, Paper 17] discovered more than 20 years later.

The characteristic function was again the foundation for the solutions proposed by von Neumann and Morgenstern for cooperative games. However, several new ideas are involved. The first of these is that of an imputation, which is a distribution of the total gain among the players of a game. For an $n$-person game, an imputation is a vector $\alpha=\left(\alpha_{1}, \cdots, \alpha_{n}\right)$ where $\alpha_{i}$ denotes the share of the $i$ th player. This share must be at least as much as he can win when playing alone and, for games in which the total amount won by the players is a constant $c, \alpha_{1}+\cdots+\alpha_{n}=c$. Otherwise, all distributions are considered as possible.

Imputations are compared by the relation of domination. An imputation $\alpha$ is said to dominate another imputation $\beta$ if there exists a coalition $S$ such that (1) $\alpha_{i}>\beta_{i}$ for $i \in S$, and (2) the amount allotted to $S$ by $\alpha$ does not exceed $v(S)$, the amount they can assure themselves in play. Thus, if $\alpha$ dominates $\beta$ and if neither is ruled out by some accepted standard of behavior, the coalition $S$ will prefer $\alpha$ to $\beta$ and has the means to enforce this preference.

With these definitions, a von Neumann-Morgenstern solution for a cooperative game is not a single imputation but rather a set $V$ of imputations which is without internal domination and is such that every imputation not in $V$ is dominated by some imputation in $V$. Any set $V$ of imputations which satisfies these two conditions of con- 
sistency and stability is called a solution. It is of interest to note that, in the 1928 solution of the zero-sum three-person game, the three imputations corresponding to the three possible coalitions form the only finite solution to this game.

In all of the $n$-person games that have been investigated thus far, solutions have been found. Indeed, the number of solutions is embarrassing in the absence of a general proof of the existence of a solution for every $n$-person game. The problem may be considered without regard to game theory, by treating domination as an abstract relation on a set of points. In [D] it is shown that, with additional conditions on the relation, a solution exists and is unique. In a series of papers [23] Moses Richardson has considerably weakened these conditions but, to this date, the problem has not been solved.

Von Neumann's interest in this question never waned and he followed closely the attempts of Shapley [15, Paper 20] and Gillies [15, Paper 19], [18] to expand the class of games for which solutions are known to exist. Any hope for regularity in the solution sets was dashed by Shapley, who has shown [18] that an $n$-person game can be constructed with a solution containing an arbitrary closed set as isolated component in an $(n-3)$-dimensional subset of the space of imputations.

At this point it seems of interest to mention some remarks made by von Neumann at Princeton, February 1, 1955, as chairman of a round-table discussion of research in $n$-person games. We quote (freely) from Philip Wolfe's report:

"Von Neumann pointed out that the enormous variety of solutions which may obtain for $n$-person games was not surprising in view of the correspondingly enormous variety of observed stable social structures; many differing conventions can endure, existing today for no better reason than that they were here yesterday. It is, therefore, still of primary importance to settle the general question of the existence of a solution for any $n$-person game. Another gap in present knowledge pertains to the formation of the equilibria constituting the solution of an $n$-person game; even the beginning of a dynamic theory is lacking.

"Von Neumann then outlined the program of a new approach to the cooperative game by means of some (not yet constructed) theory of the rules of games. A class of admissible extensions of the rules of the non-cooperative game would be defined to cover communication, negotiation, side payments, etc. It should be possible to determine when one admissible extension was 'stronger' than another, and the game correspondingly more cooperative. The goal would be to find a 'maximal' extension of rules: A set of rules such that the non-cooperative solutions for that game do not change under any stronger set. If this were possible, it would seem the ideal way in which to sulve the completely cooperative game."

Roughly two-thirds of the more than 600 pages of the von Neumann-Morgenstern treatise [D] are devoted to cooperative games. They contain a wide assortment of concepts and techniques, too 
wide to be treated with any justice here. For a balanced appraisal which relates von Neumann's work quite clearly to the later contributions it inspired, the reader is referred to the recent book of R. D. Luce and H. Raiffa [17]. This presents an invaluable survey of the current state of the Theory of Games, especially cooperative ones.

Extensive games. Throughout von Neumann's work there is evidence of a strong drive to supply rigorous axiomatic foundations for the subjects which he treated. For game theory, his contribution of this sort was a set-theoretical formulation of a very wide class of finite games. When presented in terms of this system, a game is said to be in extensive form. Although the extensive description of a game is stripped of all the technical apparatus peculiar to the particular game, it retains the full combinatorial complexity of play according to the original rules. As such, it is in contrast with the so-called normalized form, with but one choice per player, that results when the game is simplified through the introduction of pure strategies.

Von Neumann's achievements in this direction were many. First, and most important, he recognized the necessity of characterizing games of strategy in an unequivocal manner; otherwise the field would necessarily remain a mere collection of examples. This program he carried out in masterful style, so that the essential elements which he isolated, such as play, chance and personal moves, payoffs, and information partitions, are still the starting points of any description of a game. It is difficult to appreciate, in retrospect, the genius of von Neumann's abstraction of the essential elements of a general game of strategy into a mathematical system. For this area, perhaps more than any other he worked in, had little mathematical tradition. Yet the mathematical system he created has served as a rallying point for much mathematical research which otherwise would not have been possible.

He placed in bold relief the pioneering theorem that every game with perfect information has a minimax solution in pure strategies. This theorem, used implicitly by many writers, could have no general proof until the theory of extensive games was on sound footing. By repeated emphasis, he underlined the rôle of incomplete information as the source of many interesting game-theoretic results. The ability to describe rigorously the state of information at each move in a game, separating out "inferences" and "signals," and to construct strategies which take full account of this information had a decisive rôle in the development of a theory of extensive games.

It is instructive to contrast the treatments of extensive games given 
in the 1928 paper and in the 1944 treatise. Although a full discussion was not possible within the restricted length of the 1928 paper, one feels that von Neumann slighted the problem of characterizing strategic games in his zeal to reach his main goal at that time, the Minimax Theorem. However, external evidence is available to show that the problem was of interest to him in that period. It is known that von Neumann suggested to D. König [11] before 1927 the application of a graph-theoretical result to show the finiteness of a game with a stop rule and, from the same König paper, that he had corrected an error in the Zermelo proof of the pure-strategy determinateness of chess [30]. Also, a paper of L. Kalmar [8], provides the information that at the same time he had formulated and proved the minimax solution (strict determinateness) in pure strategies of the general game with perfect information.

The class of games normalized in the 1928 paper is restricted to those finite games in which a player knows, at the occasion of a choice, either everything or nothing about the anterior choices. At the urging of Morgenstern this class was widened in the 1944 treatise, through a new formulation in terms of partitions of moves, to include games in which there may be only partial information about prior choices. (The work on this chapter, as on many others in the book, continued from breakfast through evening parties. Once, exasperated with the persistence of the collaborators, Klara von Neumann declared that she would have nothing more to do with the Theory of Games unless it "included an elephant." It does, on page 64 of [D].)

By and large, subsequent work on extensive games proceeded from ideas initiated by von Neumann. Among predominant lines, one may cite the literature on games with perfect information [15, Papers $11,12,13 ; 4$, Papers 6-11] and on the concept of behavior strategies $[15$, Papers $11,14,15]$ introduced by von Neumann in his analysis of poker.

Poker. Chess, Poker and Bridge are familiar games in extensive form which exhibit three quite distinct levels of information structure. Chess is a game of perfect information; in theory, as remarked above, it possesses a strictly determined solution in pure strategies. Poker has imperfect information but perfect recall by each player; it possesses a strictly determined solution in behavior (i.e. locally randomized) strategies. Bridge has imperfect information and even imperfect recall; it may be strictly determined only in mixed (i.e. fully randomized) strategies, as shown in a highly simplified instance analyzed by G. L. Thompson [15, Paper 15].

Poker provides a superb laboratory example for game theory be- 
cause its decisive strategic characteristics, centered on "Bluffing" phenomena, can be conserved in suitably simplified variants that have extensive forms of reasonable complexity. Indeed, it seems that many of von Neumann's initial ideas on game theory were shaped by his analysis of two-person variants of Poker during the fruitful years 1926-1928 (see footnote on page 186 of [D]). This systematic analysis occupies a major portion of Chapter IV of the von NeumannMorgenstern treatise [D] as well as a lengthy supplement which D. B. Gillies and J. P. Mayberry helped prepare for belated publication [I]. There von Neumann achieved an exact theory which reduced the maneuvers of Bluffing to quantitative terms and disentangled mixed psychological motives by relating them to the main strategic features of the game. Not only do his minimax solutions of Poker variants prescribe Bluffing as a rational activity, rather than a psychic one, but they also specify, in general, definite probabilities with which Bluffing should be employed at each opportunity. This was a major "break-through" which even the layman may be expected to appreciate, judging by various magazine articles and a fine popular book [19].

An important technical feature of von Neumann's work on Poker was his successful determination of minimax solutions through use of locally randomized strategies, now known as behavior strategies. Later research [15, Paper 11] has shown that such randomization, usually much simpler than overall randomization by mixed strategies, is sufficient to determine strictly the minimax solution of any game with perfect recall. Another technical feature was the replacement for computational convenience of the finite discrete scale of Poker hands by an infinite linear continuum. This seems to have been von Neumann's only venture into infinite or continuous games. However, since his Poker games were not in normalized form, their infinite nature had no direct bearing on the abundant work in infinite games carried on by Ville [26], Wald [28], and others (see for example [14, Papers 12-15; 15, Papers 6-10]). (Von Neumann closed the 1955 round-table discussion mentioned above with the joking remark, "Don't blame me for infinite games!")

An additional instance of the value of Poker as a laboratory example is the three-person Poker variant analyzed by J. F. Nash and L. S. Shapley [14, Paper 10]. This helped shape the Nash theory of equilibrium points for noncooperative $n$-person games [20].

Utility. The fundamental problem of the theory of games is to find the methods by which a player can obtain a "most favorable result." At first, von Neumann identified the "most favorable result" with 
the greatest expected (monetary) value [A, p. 298], remarking that this or some similar assumption was necessary in order to apply the methods of probability theory. While doing so, he was well aware of the objections to the principle of maximizing expected winnings as a prescription for behavior, but wished to concentrate on other problems. To state some of these objects and the contributions made by von Neumann when he returned to the problem, consider the following situation: Let a player be required to choose an element $x$ from a set $X$ and let $f(x)$ be the monetary payoff resulting from this act. If the player always prefers more money to less, then the rule for behavior is clear in this situation of certainty, namely, choose $x$ so as to maximize $f(x)$ if possible.

If this situation is now altered so as to introduce risk, say by enlarging $X$ to the set of all probability distributions on $X$, the rule is no longer obvious. If the index $f(x)$ is extended to probability mixtures by setting it equal to the mathematical expectation $E(f(x))$, the principle of maximizing expected winnings does not reflect the preferences of many people. To express the classical example of the St. Petersburg paradox in these terms, set $X=\{0,1, \cdots, n, \cdots\}$ with $f(0)=0$ and $f(n)=2^{n}-c$ for $n>0$, where $n=0$ represents the status quo and $c$ stands for the entry fee. The rule of maximizing expected winnings prefers the probability distribution $p_{n}=1 / 2^{n}$ for $n>0$ to the status quo $n=0$ with certainty, no matter how large the entry fee $c$. However, numerical examples persuade many people of the advantages of the status quo; for example, with $c=\$ 128$, there is but one chance in 64 that a player who chooses the probability mixture will break even, and he will otherwise lose at least $\$ 64$. If the game is to be played but once, only the most foolhardy (or wealthy) would assume this risk for the prospect of highly improbable winnings.

To resolve this paradox, Daniel Bernoulli suggested that people do not follow monetary value as an index for preferences, but rather the "moral worth" of the money. Furthermore, in a situation involving risk, they seek to maximize the expected value of moral worth, or what has been called "moral expectation." Finally, he proposed a quite serviceable function to measure the moral worth of an amount of money, namely, its logarithm.

Whatever the defects of this function as a universal measure of preferences, and they are many, it raises the question of the existence of a numerical index which will reflect accurately the choices of an individual in situations of risk. Interest in this problem as posed was first shown by F. P. Ramsey [22] who went beyond Bernoulli in 
that he defined utility operationally in terms of individual behavior. (Once von Neumann was asked why he did not refer to the work of Ramsey, which might have been known to someone conversant with the field of logic. He replied that, after Gödel published his papers on undecidability and the incompleteness of logic, he did not read another paper in symbolic logic.) Independently, von Neumann confronted this problem with Morgenstern in [D], while they were laying the bases for the theory of games. Informally, they showed that if a player is able to express his preferences between every possible pair of probability mixtures of outcomes in a consistent manner, then there exists a utility function defined on the possible outcomes, the expected value of which can be used as a guide to the player's choices in situations of risk. Precisely, they proved the following theorem.

Let $X=\{x, y, z, \cdots\}$ be a set on which a preference relation $>$ is defined satisfying:

(1) For all $x$ and $y$ in $X$, exactly one of the following holds: $x=y, x>y, y>x$.

(2) If $x>y$ and $y>z$ then $x>z$.

Let an operation be defined for all $x$ and $y$ in $X$ and all real numbers $\alpha, 0<\alpha<1$, yielding $\alpha x+(1-\alpha) y$, an element of $X$ and satisfying:

(3) $\alpha x+(1-\alpha) y=(1-\alpha) y+\alpha x$.

(4) $\alpha(\beta x+(1-\beta) y)+(1-\alpha) y=(\alpha \beta) x+(1-\alpha \beta) y$.

Here + is part of a formal operational symbol. Let the operation and the preference relation jointly satisfy:

(5) If $x>y$ then $x>\alpha x+(1-\alpha) y>y$ for all $\alpha$.

(6) If $x>y>z$, then there exist $\alpha$ and $\beta$ such that $\alpha x+(1-\alpha) z>y$ $>\beta x+(1-\beta) z$.

Then there exists a real valued function $u$ defined on $X$ and satisfying:

(7) If $x>y$ then $u(x)>u(y)$.

(8) For all $x, y$ and $\alpha, u(\alpha x+(1-\alpha) y)=\alpha u(x)+(1-\alpha) u(y)$. This utility function is unique to a positive linear transformation.

The proof of this theorem appears in the second and third editions of [D]. Although von Neumann was never satisfied with the form of this proof, a detailed analysis of cases, it was available at the time of the first edition and was added at the insistence of Morgenstern ${ }^{2}$ who found that economists were not convinced that a rigorous proof could be given.

The reexamination of the utility concept by von Neumann and

2 Professor Morgenstern reports that the article of Karl Menger, Das Unsicherheitsmoment in der Wertlehre, Z. Nationalökonomie vol. 5 (1934) pp. 459-485, played a primary role in persuading von Neumann to undertake a formal treatment of utility. 
Morgenstern stimulated activity in this area by economists, statisticians, and mathematicians. An excellent review of the economic literature is given by Arrow [1]. The point of view of subjective probability is set forth by Savage [24]. New axiom systems for utility theory have been developed by Hausner [5] and Herstein and Milnor [6].

Economic equilibrium. Parallel to his interest in the mathematical treatment of competitive economic situations by means of games of strategy, von Neumann was concerned with the formulation of models of general economic equilibrium. In 1932, he presented an economic model of his own devising at a colloquium in Princeton. This paper (published in $[\mathbf{B}]$ and translated in $[\mathbf{C}]$ ), together with work of Wald [27] marks a new era in mathematical economics in which rigorous arguments replace mere "equation counting" as a means of establishing the existence of economic equilibrium. It also shares with Wald's work the credit for recognizing explicitly that inequalities as well as equations are forced on the system by the economic context, and for utilizing this fact to advantage in the mathematical treatment. In its linear approach to production, with alternative processes and the possibility of intermediate goods, it is the direct ancestor of linear programming and activity analysis.

The system is a closed pure production model in which $n$ goods are produced by $m$ processes in discrete time periods, with the outputs of one period the inputs of the next. Constant returns to scale are assumed as well as the unlimited availability of natural materials of production. The problem is: given $m$ by $n$ matrices $A$ and $B$ whose nonnegative elements $a_{i j}$ and $b_{i j}$ specify the amounts of the $j$ th good consumed and produced by the $i$ th process operating at unit intensity over one time period, it is required to find the intensity (row) vector $x$, the price (column) vector $y$, the expansion factor $\alpha$ and the interest factor $\beta$ that together make for economic equilibrium. The $m$ nonnegative components of $x$ are the intensities at which the various processes are operated, the $n$ components of $x B$ the resulting amounts of the various goods produced in a given time period, and the $n$ components of $\alpha x A$ the resulting amounts of the various goods consumed in the next period. Since the economy is "closed," it is required that

$$
x B-\alpha x A \geqq 0 .
$$

If a good is overproduced, its price is to be zero. The $n$ nonnegative components of $y$ are the prices assigned to the various goods, the $m$ components of $B y$ the resulting revenues from the various processes in a given time period, and the $m$ components of $\beta A y$ the resulting 
input costs for the various processes in the next period. Since the economy is "profitless," it is required that

$$
B y-\beta A y \leqq 0 .
$$

If an activity operates at a loss, its intensity is to be zero.

To ensure that the economy does not decompose into groups of goods without interactions, von Neumann assumed that every good appears in every activity, either as an input or output, i.e.

$$
A+B>0 \text {. }
$$

With this condition, he showed that a uniformly expanding economy is possible and that the factors of expansion and of interest are necessarily equal.

The relations between this theorem and the Minimax Theorem are close and somewhat unexpected. If the intensity and price vectors $x$ and $y$ are both normalized so that components sum to one, they form probability vectors which may be regarded as mixed strategies for the players of a zero-sum two-person game with payoff matrix $B-\lambda A$. The existence of an equilibrium with a unique common value for $\alpha$ and $\beta$ is then merely the assertion that there is a unique choice of $\lambda(=\alpha=\beta)$ which makes this a "fair" game (i.e. value zero). This fact is established on the basis of von Neumann's assumption.

At the same time, the equilibrium conditions give rise to a minimax saddlepoint of

$$
x B y / x A y,
$$

the ratio of total revenue to total cost. The saddlepoints of this function reappear with a different interpretation in the work of L. S. Shapley on stochastic games [25].

The economic assumptions of this originative model have been reexamined recently by David Gale [16, Paper 18], and by J. G. Kemeny, Oskar Morgenstern, and G. L. Thompson [9]. In independent work, they have relaxed the requirement $A+B>0$ and thus are led into a discussion of the decomposition of the economic system and the possibility of nonunique factors of expansion and interest. In the latter paper, the connection with game theory is thoroughly explored.

\section{Part II. Minimax Methods and Computation}

Minimax Theorem. Let an arbitrary real $m$ by $n$ matrix $A$ be given as the "payoff matrix" of a zero-sum two-person game in normalized form. Let $x$ and $y$ be probability vectors providing mixed strategies for the two players of the game: $x$ is any row of $m$ non- 
negative components summing to one and $y$ any column of $n$ nonnegative components summing to one. Then the mappings

(1) $x \rightarrow \min x A$ (=minimum component of $x A$ ),

(2) $y \rightarrow \max A y$ (= maximum components of $A y$ ),

(3) $(x, y) \rightarrow x A y$

define three continuous functions from compact convex sets into the real numbers; (1) is convex piecewise-linear, (2) is concave piecewiselinear, and (3) is bilinear. In (1) $\min x A$ is the "floor" under the expected gains of Player I resulting from his mixed strategy $x$; in (2), $\max A y$ is the "ceiling" over the expected losses of Player II resulting from his mixed strategy $y$; and in (3), $x A y$ is the expected value of the payoff resulting from $x$ and $y$. The three functions are related by a combination of identities and inequalities

$$
\min x A=\min _{y} x A y \leqq x A y \leqq \max _{x} x A y=\max A y .
$$

In his fundamental Minimax Theorem (the Main Theorem of the Theory of Games) von Neumann established the existence of a unique value $v$ such that

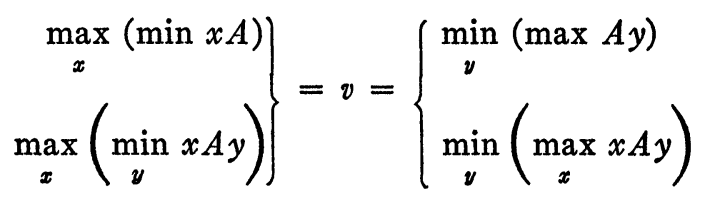

and some optimal (or good) mixed strategies $x^{0}$ and $y^{0}$ such that

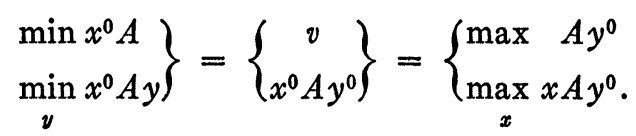

Thus

(1) $v=\min x^{0} A$ is the maximum "floor" for Player I,

(2) $v=\max A y^{0}$ is the minimum "ceiling" for Player II,

(3) $v=x^{0} A y^{0}$ is the saddlevalue of the expected payoff.

A "solution" $\left(x^{0}, y^{0}, v\right)$ of the "matrix game" consists of $m+n+1$ components which can be characterized algebraically as a solution of the $2 m+2 n$ linear inequalities

$$
x^{0} \geqq 0, \quad A y^{0} \leqq v, \quad y^{0} \geqq 0, \quad x^{0} A \geqq v
$$

and 2 linear equations

$$
x_{1}^{0}+\cdots+x_{m}^{0}=1, \quad y_{1}^{0}+\cdots+y_{n}^{0}=1 .
$$

Analytic minimax methods. The analytic proofs of the Minimax Theorem given by von Neumann were of two essentially different 
types. Proofs of the first type (see $[\mathrm{A}]$ and $[\mathrm{B}]$ ) are based explicitly on extensions of the Brouwer fixed point theorem; proofs of the second type (see $[\mathbf{F}],[\mathbf{G}]$, and $[\mathbf{K}]$ ), while related implicitly to fixed point equilibria, are cast either in terms of differential equations or iterative approximation algorithms.

An immediate connection between the Minimax Theorem and the fixed points of point-to-set functions may be seen in the following situation. Given a zero-sum two-person game with payoff matrix $A$, define

$$
\begin{aligned}
& F(x)=\left\{y^{0} \mid x A y^{0}=\min _{y} x A y\right\}, \\
& G(y)=\left\{x^{0} \mid x^{0} A y=\max _{x} x A y\right\} .
\end{aligned}
$$

These sets may be called the strategies counter to $x$ and $y$, respectively. Then, either of the following statements is equivalent to the equality $\max _{x}\left(\min _{y} x A y\right)=\min _{y}\left(\max _{x} x A y\right)$ :

(a) There exists a strategy $x^{0}$ such that $x^{0} \in G\left(F\left(x^{0}\right)\right)$.

(b) There exist strategies $x^{0}$ and $y^{0}$ such that

$$
\left(x^{0}, y^{0}\right) \in G\left(y^{0}\right) \times F\left(x^{0}\right) .
$$

These may be paraphrased by: (a) there exists a strategy $x^{0}$ countering a strategy $y^{0}$ which is counter to $x^{0}$, and (b) there exist strategies $x^{0}$ and $y^{0}$ countering each other. Either of these statements asserts the existence of a fixed point of a point-to-set mapping.

Von Neumann developed the necessary fixed point theorems in two stages. The 1928 paper contains the following result [A, pp. 310311]: Let $H(x)$ be a continuous ${ }^{3}$ point-to-set mapping defined on $0 \leqq x \leqq 1$ and with closed subintervals as values. Then there exists $x^{0}$ such that $x^{0} \in H\left(x^{0}\right)$.

To insure the convexity of the image sets, the notion of quasiconvexity was used. (A real valued function $\phi$ defined on a (convex) set $Y$ in the affine space $A^{n}$ is said to be quasiconvex if the "level sets" $L_{a}=\{y \mid \phi(y) \leqq a\}$ are convex for all $a$; if $-\phi$ is quasiconvex, then $\phi$ is said to be quasiconcave.) Von Neumann proved [A, p. 309]: Let $f(x, y)$ be a continuous real valued function defined on $0 \leqq x \leqq 1$ and $0 \leqq y \leqq 1$. If $f(x, y)$ is quasiconvex in $y$ for each $x$ and quasiconcave in $x$ for each $y$, then

$$
\max _{x} \min _{y} f(x, y)=\min _{\nu} \max _{x} f(x, y) .
$$

${ }^{8}$ Recall that a point-to-set mapping is continuous if its graph is closed. 
That is, a continuous game on the square with payoff quasiconvex in $y$ and quasiconcave in $x$ always has a saddlepoint in pure strategies. The full implications of this result were only realized in the work [14, Paper 15] of H. F. Bohnenblust, S. Karlin, and L. S. Shapley. A minimax theorem with similar assumptions has also been proved by H. Kneser [10].

The use of the weaker assumption of quasiconvexity and quasiconcavity (rather than the actual bilinearity of the payoff of a zerosum two-person game) permitted the proof of the following stronger theorem by successive projections [A; p. 307]: Let $f(x, y)$ be a continuous real valued function defined on $X \times Y$, where $X$ and $Y$ are non-empty compact convex subsets of the affine spaces $A^{m}$ and $A^{n}$, respectively. If $f(x, y)$ is quasiconvex in $y$ for each $x \in X$ and is quasiconcave in $x$ for each $y \in Y$, then

$$
\max _{x} \min _{y} f(x, y)=\min _{y} \max _{x} f(x, y) .
$$

The application of this theorem to the special case of zero-sum twoperson games is clear.

For the analysis of his expanding economy model, von Neumann extended his fixed point result as follows [B, p. 80]: Let $F: X \rightarrow Y$ and $G: Y \rightarrow X$ be continuous point-to-set mappings defined on $X$ and $Y$, non-empty compact convex subsets of the affine spaces $A^{m}$ and $A^{n}$, and taking as values compact convex subsets of $Y$ and $X$, respectively. Then there exist $x^{0}$ and $y^{0}$ such that $\left(x^{0}, y^{0}\right) \in G\left(y^{0}\right)$ $\times F\left(x^{0}\right)$.

Taking $Y=X$ and $G(y)=y$ for all $y \in Y$, we have the formulation of S. Kakutani [7]: Let $F(x)$ be a continuous point-to-set mapping defined on $X$, a non-empty compact subset of $A^{m}$, and taking as values compact convex subsets of $X$. Then there exists an $x^{0}$ such that $x^{0} \in F\left(x^{0}\right)$.

In view of von Neumann's deep involvement with the development of high speed automatic computing machinery during the postwar years, it is not surprising that his work on the computational problems of game theory aimed at practical numerical methods to solve large games on digital computers. When he made his first contribution to this area in 1948, most of the machines now widely available were in planning stages, or at best, in the earliest stages of construction. With this in mind, and considering our ignorance of the types of games (and linear programs) that would later arise for solution, von Neumann insisted that any algorithm should be accompanied by a rigorous upper bound on the number of steps, valid for all matrix games. 
By 1948, several abstract proofs of the Minimax Theorem were known. It is clear in retrospect that each of these can be transformed with very little difficulty into an explicit algorithm for the computation of the value of the game and its optimal strategies. If the number of alternatives for the two players are $m$ and $n$ with $m \leqq n$, then the number of steps required will be some function of $n$, say $f(n)$, which goes to infinity with $n$. Methods which mirror the existence proofs entail computations with $f(n)$ the order of $2^{n}$ or $n$ !, which are clearly impractical for machine computation when $n$ is even moderately large.

Von Neumann often expressed the opinion that there are (primarily heuristic) reasons to expect that the optimum $f(n)$ would be a good deal smaller. Pointing out the parallel situation with regard to linear equations, and emphasizing that the solution of a matrix game has, as a saddlepoint problem, more stability than a system of linear equations, he conjectured on several occasions that the optimum $f(n)$ would have the order of $n^{3}$.

The algorithm proposed in [F] leads to approximate solutions by an iterative procedure. To describe the essentials of this method, which has never been published, let $A=\left(a_{i j}\right)$ be the $m$ by $n$ payoff matrix of a zero-sum two-person game which has been scaled so that $-1 \leqq a_{i j} \leqq 1$ for all $i$ and $j$. Positive constants $\theta$ and $\zeta$ being given, the first stage will be to compute either a mixed strategy $x$ such that (1) $\min x A>-\theta$, or a mixed strategy $y$ such that (2) $\max A y<\zeta$. If (2) holds for a given $y$, then this stage ends. Otherwise, form the vector $u=\max (A y, 0)$ from the positive components of $A y$. Then $x=u / \sum_{i} u_{i}$ is a mixed strategy for the first player. If (1) holds for this $x$, then this stage ends. Otherwise, pick $j_{0}$ such that $\sum_{i} x_{i} a_{i j_{0}}$ $\leqq-\theta$ and set

$$
\epsilon=-\sum_{i} x_{i} a_{i j_{0}} / \sum_{i} a_{i j_{0}}^{2}
$$

Define $y^{\prime}$ by

$$
(1+\epsilon) y_{j}^{\prime}=\left\{\begin{array} { l } 
{ y _ { j } + \epsilon } \\
{ y _ { j } }
\end{array} \text { for } \left\{\begin{array}{l}
j=j_{0} \\
j \neq j_{0} .
\end{array}\right.\right.
$$

(This procedure may be described informally as forming a mixed strategy for your opponent that weights his pure strategies in proportions equal to your losses above a fixed level. The relative weight of your pure strategy that is best against this fictitious mixed strategy for your opponent is then increased. The choice of $\epsilon$, which determines this increase in weight, minimizes a measure of the losses incurred by $y^{\prime}$ which is closely related to $\sum_{i} u_{i}^{\prime 2}$.) 
Von Neumann obtained

$$
\frac{\log \left(m n \zeta_{1}^{2} / \zeta^{2}\right)}{-\log \left(1-\theta^{2} / m\right)}+1 \quad\left(\text { where } \zeta_{1}=\max A y\right)
$$

as a bound on the number of times this procedure may have to be repeated before either (1) or (2) holds. The interval $(-\theta, \zeta)$ is then narrowed and the next stage begins.

The final estimate of the time required for this method, for a matrix scaled to lie between 0 and 1 , is that the total number of multiplications required for an accuracy of $\gamma$ in the value is majorized by $17.56 m^{2} n \gamma^{-2} \log (m n)$. Thus allowing one millisecond for a multiplication, to obtain the value of a 100 by 200 game to an accuracy of $10 \%$ would require not more than 400 days of computation. As impractical as this may seem, it is better than any other generally valid bound obtained to this date.

In the joint paper [G] with G. W. Brown, von Neumann advanced a related procedure in the form of differential equations. Working with a symmetric game having a skew-symmetric payoff matrix $A=\left(a_{i j}\right)$ and a mixed strategy $y$, take $u=\max (A y, 0)$ as before. Set $\phi(y)=\sum_{i} u_{i}$. Then the differential equation system

$$
\frac{d y_{i}}{d t}=u_{i}-\phi(y) y_{i}
$$

has a unique solution (for any initial conditions) with limit points $y^{\infty}$ which solve $A$. These equations find a discrete analogue in the Brown method of "fictitious play" [12, Chapter 24] and are closely related to the mapping used by J. Nash [20] to prove the Minimax Theorem by a direct application of the Brouwer fixed point theorem.

In his last published work [K] on game theory, von Neumann presented a third iterative method for solving zero-sum two-person games. The procedure applies to a triplet $(x, y, k)$ consisting of two mixed strategies and a real number. Let $u=\max (A y-k, 0)$ and $v=\max (k-x A, 0)$. Then $\psi(x, y, k)=\sum_{i} u_{i}^{2}+\sum_{j} v_{j}^{2} \geqq 0$ provides an index of the failure of the triplet to be a solution; $\psi=0$ if and only if $(x, y, k)$ is a solution. A new triplet is defined as a weighted average of $(x, y, k)$ with a triplet $(\bar{x}, \bar{y}, \bar{k})$, where

$$
\bar{x}=u / \sum_{i} u_{i}, \quad \bar{y}=v / \sum_{j} v_{j}, \quad \text { and } \quad \bar{k}=\bar{x} A \bar{y}
$$

(if $u=0$, take any mixed strategy for $\bar{x}$; if $v=0$, take any mixed strategy for $\bar{y}$ ). The weighting is chosen to make $\psi$ small for the new triplet. After $h$ steps, it can be shown that 


$$
\psi \leqq \frac{m+n}{h}\left[\max _{i, j} a_{i j}-\min _{i, j} a_{i j}\right]
$$

and hence $\psi \rightarrow 0$ as $h \rightarrow \infty$. Again, there is provided a careful and generally valid bound on the time required for a given accuracy.

Algebraic methods-Duality. In contrast with the fixed-point and gradient methods just discussed above, there are more elementary means, essentially algebraic in nature, of proving the Minimax Theorem and computing solutions of matrix games. These involve systems of linear inequalities and the related vector-space geometry of convex closed polyhedral sets. In the Theory of games and economic behavior [D, Chapter 3] von Neumann and Morgenstern develop the Minimax Theorem by a linear-convex algebraic approach initiated by Jean Ville [26]. (Oskar Morgenstern has told us that he drew Ville's article to von Neumann's attention after seeing it quite by chance while browsing in the library of the Institute for Advanced Study. They decided at once to adopt a similar elementary procedure, trying to make it as pictorial and simple to grasp as possible.)

The key result in their algebraic approach is The Theorem of the Alternative for Matrices:

For any (real) rectangular matrix $A$, there exists

either a probability vector (row) $x$ such that $x A>0$

or a probability vector (column) $y$ such that $A y \leqq 0$.

In particular, if $A$ is skew-symmetric, there must exist a probability vector $x$ for which $x A \geqq 0$. This establishes the Minimax Theorem for a symmetric game, in which the payoff matrix is skew-symmetric and the game value zero. For a game with arbitrary payoff matrix the Minimax Theorem follows from the Alternative Theorem by various devices, such as symmetrization (discussed below) or translation into a "fair" game by adding a suitable constant (negative or positive) to all payoffs.

It is now known that the Alternative Theorem falls within the Transposition Theorem of Motzkin and is closely related to classical results of Farkas, Gordan and Stiemke. These theorems can be proved by common rational algebraic means and have the same inherent duality of negative-transpose nature that a game payoffmatrix possesses [16, Paper 1]. For example, a sharpened form of the Alternative Theorem can be stated as follows. Let $A$ be an arbitrary rectangular matrix over an ordered field and $B$ its negative transpose $\left(B=-A^{T}\right)$. Then the dual systems

$$
x A \geqq 0, x \geqq 0 \quad \text { and } \quad y B \geqq 0, y \geqq 0
$$


possess solutions $x^{*}$ and $y^{*}$ such that

$$
x^{*} A+y^{*}>0 \text { and } y^{*} B+x^{*}>0 .
$$

In particular, if $A$ is skew-symmetric (i.e. $A=B$ ), then the self-dual system $x A \geqq 0, x \geqq 0$ possesses a solution $x^{*}$ such that $x^{*} A+x^{*}>0$.

As suggested above, the Minimax Theorem can be proved by rational algebraic means and holds for any ordered field. This was first specifically pointed out and carried through by Hermann Weyl [14, Paper 2]. Others [14, Paper 7] achieved the same end by showing that the solution of a game with arbitrary $m$ by $n$ payoff matrix $A$, easily translated to have positive value, was equivalent to the solution of a symmetric game with the skew-symmetric payoff matrix of order $m+n+1$

$$
\left[\begin{array}{crr}
0 & A & -1 \\
-A^{T} & 0 & 1 \\
1 & -1 & 0
\end{array}\right] .
$$

Earlier von Neumann [G, p. 76] had indicated a more natural symmetrization leading to an equivalent symmetric game of order $m n$.

In an unpublished working-paper [E] von Neumann indicated that the (analytic) problem of maximizing a linear function

$$
c y=c_{1} y_{1}+\cdots+c_{n} y_{n}
$$

constrained by $m+n$ linear inequalities

$$
A y \leqq b, \quad y \geqq 0
$$

is equivalent to the (rational algebraic) problem of solving a system of $2 m+2 n+1$ linear inequalities in $x$ and $y$ :

$$
x \geqq 0, \quad A y \leqq b, \quad y \geqq 0, \quad x A \geqq c, \quad c y \geqq x b .
$$

This led directly into the duality and existence theorems of Linear Programming [12, Chapter 19] as well as relations with a "fair" game having the payoff matrix

$$
\left[\begin{array}{rr}
A & -b \\
-c & \delta
\end{array}\right] \quad(\text { where } \delta=\max c y) \text {. }
$$

Treating the "dual variables" $(x)$ as nonnegative Lagrange multipliers, the authors [13] went on to get analogous conditions, both necessary and sufficient, for maximizing a concave function constrained by suitable concave inequalities.

It was observed by G. B. Dantzig [12, Chapter 20] and G. W. 
Brown that the above system of $2 m+2 n+1$ linear inequalities could be written as

$$
\left[x, y^{T}, 1\right]\left[\begin{array}{ccc}
0 & A & -b \\
-A^{T} & 0 & c^{T} \\
b^{T} & -c & 0
\end{array}\right] \geqq 0, \quad\left[x, y^{T}, 1\right] \geqq 0 .
$$

Since this composite matrix is skew-symmetric, it can be taken as the payoff matrix of a symmetric game. (Cf. the composite matrix above, in which $A$ is the payoff matrix of a positively-valued game, $b=1$ and $c=1$.) Thus, the solution of a pair of dual linear programs, if both are feasible, can be related to the solution of a symmetric game.

The duality between opponents in a matrix game and between dual linear programs bears striking resemblance to the duality in the von Neumann economic model $[\mathbf{B} ; \mathbf{C}]$, discussed above, between the intensity vector $x$ and expansion factor $\alpha$ on the one hand and the price vector $y$ and the interest factor $\beta$ on the other. However, the solution of the economic model cannot be achieved in general by rational algebraic means; a solution with irrational components can arise from rational matrices $A$ and $B$. It is interesting to note that a similar possible irrationality goes with Nash's equilibrium-point generalization of the minimax solution of a matrix game.

Combinatorial methods. In the last years of his life, which were filled by service to his country, von Neumann found little time to pursue his many ideas relative to the Theory of Games. A new theorem or computational method might lie for years in his files, or be communicated only in conversations with colleagues. A happy exception is provided by his paper on a matrix game equivalent to the personnel assignment problem $[\mathbf{H}]$, a paper that opened a whole new area of application of linear programming and game theory to combinatorial problems. This paper was produced by inviting von Neumann to deliver his result to the Princeton seminar in game theory and then presenting him with notes taken by a member of the seminar to be edited for publication. The scheme worked, due to von Neumann's cheerful cooperation, and thus this influential paper appeared in print.

The personnel assignment problem calls for the assignment of $n$ men to $n$ jobs, one man to each job, so as to make the sum of the rated values of the man-job pairings as large as possible. More specifically, given an $n$ by $n$ matrix $R=\left(r_{i j}\right)$, where $r_{i j}$ is the positive numerical rating of the $i$ th man in the $j$ th job, the problem is to find an $n$th order permutation matrix $P=\left(p_{i j}\right)$ that will maximize 


$$
\sum_{i, j} p_{i j} r_{i j}
$$

This problem is classically trivial since it involves just a finite set of $n$ ! possibilities. But in the modern age of automatic highspeed computers it takes on real meaning; the aim is an algorithm that will be practical in terms of time and equipment available.

Von Neumann suggested that the $n$ ! possible permutation matrices $P=\left(p_{i j}\right)$ be plotted as points $X=\left(x_{i j}\right)$ in an $n^{2}$-dimensional space. Then the problem becomes that of maximizing the linear form

$$
R(X)=\sum_{i, j} r_{i j} x_{i j}
$$

on the set of $n$ ! points $P$. Extending this finite set of points to its convex hull makes no essential change in the maximization problem and translates a discrete situation into a continuous one, which becomes the following linear program: to maximize the linear form $R(X)$ constrained by

$$
x_{i j} \geqq 0 \quad \text { and } \quad \sum_{j} x_{i j}=1=\sum_{i} x_{i j} \quad(i, j=1, \cdots, n) .
$$

Proceeding further, von Neumann proved that the minimax solution of the following game of Hide-and-Seek is equivalent to those of both the preceding problems: Player I "hides" in a cell $(i, j)$ of an $n$ by $n$ square array. Player II "seeks" I either in a row $i$ or a column $j$. If $I$ is found, he must pay II the amount $1 / r_{i j}$; otherwise the payoff is zero.

While von Neumann viewed the paper as primarily a contribution to the computational solution of the assignment problem, by opening it to attack by methods devised for matrix games and linear programs, the influence of the paper far transcends this. Already several papers [16, Papers 10-14] have followed on, exploring the relations between linear programming and systems of distinct representatives, making applications to other discrete problems, and finally unraveling the integral-solution properties peculiar to transportation problems (of which the assignment problem is but a special case).

\section{CONCLUSION}

The impact of von Neumann's Theory of Games extends far beyond the boundaries of this subject. By his example and through his accomplishments, he opened a broad new channel of two-way communication between mathematics and the social sciences. These sciences were fortunate indeed that one of the most creative mathe- 
maticians of the twentieth century concerned himself with some of their fundamental problems and constructed strikingly imaginative and stimulating models with which to attack their problems quantitatively. At the same time, mathematics received a vital infusion of fresh ideas and methods that will continue to be highly productive for many years to come. Von Neumann's interest in "problems of organized complexity," so important in the social sciences, went hand in hand with his pioneering development of large-scale high-speed computers. There is a great challenge for other mathematicians to follow his lead in grappling with complex systems in many areas of the sciences where mathematics has not yet penetrated deeply.

It was von Neumann's philosophy $y^{5}$ that the mathematician may choose to work in any of a wide variety of fields, and that the selection of working material and the resulting measure of success are largely influenced by aesthetic values. However, he warned that mathematics loses much of its creative drive when too far removed from empirical sources. This philosophy is exemplified in a most brilliant and enduring way by the Theory of Games. It is a tribute to his great genius that in his 1928 paper [A] he built such a sound and comprehensive mathematical foundation for games of strategy, an area almost new to mathematics, that he was able to see it explode in his lifetime into a broad and influential field of research.

\section{BIBLIOGRAPHY I \\ JOHN VON NEUMANN'S WORK}

in the Theory of Games and Mathematical Economics

A. Zur Theorie der Gesellschaftsspiele, Math. Ann. vol. 100 (1928) pp. 295-320.

B. Über ein ökonomisches Gleichungssystem und eine Verallgemeinerung des Brouwerschen Fixpunktsatzes, Ergeb. eines Math. Koll. Vienna (ed. by Karl Menger) vol. 8 (1937) pp. 73-83.

C. A model of general economic equilibrium, Rev. Economic Studies vol. 13 (1945-1946) pp. 1-9 (Translation of [B]).

D. Theory of games and economic behavior (with Oskar Morgenstern) 1st ed., Princeton, 1944, 625 pp.; 2d ed., Princeton, 1947, 641 pp.; 3d ed., Princeton, 1953, $641 \mathrm{pp}$.

E. Discussion of a maximum problem (unpublished working paper, Princeton, November, 1947, 9 pp.).

F. A numerical method for determination of the value and the best strategies of a zero-

4 An apt designation of Warren Weaver, Science and complexity, Amer. Scientist vol. 36 (1948) pp. 536-544.

'See von Neumann's essay "The Mathematician" in The Works of the Mind Chicago 1947, pp. 180-196 [reprinted in The World of Mathematics (J. R. Newman, ed.) New York, 1956, pp. 2053-2063]. 
sum two-person game with large numbers of strategies, mimeographed, Princeton, May 1948, 23 pp.

G. Solutions of games by differential equations (with G. W. Brown), Contributions to the Theory of Games, vol. I (ed. by H. W. Kuhn and A. W. Tucker) Ann. of Math. Studies, no. 24, Princeton, 1950, pp. 73-79.

H. A certain zero-sum two-person game equivalent to the optimal assignment problem, Contributions to the Theory of Games, vol. II (ed. by H. W. Kuhn and A. W. Tucker) Ann. of Math. Studies, no. 28, Princeton 1953, pp. 5-12.

I. Two variants of poker (with D. B. Gillies and J. P. Mayberry), ibid. pp. 13-50.

J. Communication on the Borel notes, Econometrica vol. 21 (1953) pp. 124-125.

K. A numerical method to determine optimum strategy, Nav. Res. Logist. Quart. vol. 1 (1954) pp. 109-115.

\section{BIBLIOGRAPHY II \\ OTHER REFERENCES}

1. K. J. Arrow, Alternative approaches to the theory of choice in risk-taking situations, Econometrica vol. 19 (1951) pp. 404-437; Utilities, attitudes, choices: A review note, ibid. vol. 26 (1958) pp. 1-23.

2. C. Berge, Théorie gênérale des jeux à n personnes (Mémor. Sci. Math., no. 138) Paris, 1957.

3. E. Borel, La thêorie du jeu et les équations intégrales d noyau symétrique gauche, C. R. Acad. Sci. Paris vol. 173 (1921) pp. 1304-1308; Sur les jeux ou interviennent l'hasard et l'habileté des joueurs, Theorie des Probabilités, Paris, 1924, pp. 202-224; Sur les systèmes de formes linéaires d déterminant symétrique gauche et la théorie générale $d u$ jeu, C. R. Acad. Sci. Paris vol. 184 (1927) pp. 52-54. [These three notes are reprinted, in English translation by L. J. Savage, in Econometrica vol. 21 (1953) pp. 97-117.]

4. M. Dresher, A. W. Tucker, and P. Wolfe (editors), Contributions to the Theory of Games, vol. III, Ann. of Math. Studies, no. 39, Princeton, 1957.

5. M. Hausner, Multidimensional utilities, Decision processes (R. M. Thrall, C. H. Coombs, and R. L. Davis, editors) New York, 1954, pp. 167-180.

6. I. N. Herstein and J. W. Milnor, An axiomatic approach to measurable utility, Econometrica vol. 21 (1953) pp. 291-297.

7. S. Kakutani, $A$ generalization of Brouwer's fxxed point theorem, Duke Math. J. vol. 8 (1941) pp. 457-459.

8. L. Kalmar, Zur Theorie der abstrakten Spiele, Acta Sci. Math. Szeged. vol. 4 (1928-1929) pp. 65-85.

9. J. G. Kemeny, O. Morgenstern and G. L. Thompson, $A$ generalization of the von Neumann model of an expanding economy, Econometrica vol. 24 (1956) pp. 115-135.

10. H. Kneser, Sur un théorème fondamental de la théorie des jeux, C. R. Acad. Sci. Paris vol. 234 (1952) pp. 2418-2420.

11. D. König, Über eine Schlussweise aus dem Endlichen ins Unendliche, Acta Sci. Math. Szeged. vol. 3 (1927) pp. 121-130.

12. T. C. Koopmans (editor), Activity analysis of production and allocation, Cowles Commission Monograph, no. 13, New York, 1951.

13. H. W. Kuhn and A. W. Tucker, Nonlinear programming, Proceedings of the Second Berkeley Symposium on Mathematical Statistics and Probability (J. Neyman, ed.), Berkeley, 1951, pp. 481-492.

14. H. W. Kuhn and A. W. Tucker (editors), Contributions to the theory of games, vol. I, Ann. of Math. Studies, no. 24, Princeton, 1950. 
15. Contributions to the theory of games, vol. II, Ann. of Math. Studies, no. 28, Princeton, 1953.

16. - Linear inequalities and related systems, Ann. of Math Studies, no. 38, Princeton, 1956.

17. R. D. Luce and H. Raiffa, Games and decisions, New York, 1957.

18. R. D. Luce and A. W. Tucker (editors), Contributions to the theory of games, vol. IV, Ann. of Math. Studies, no. 40, Princeton (in press).

19. J. McDonald, Strategy in poker, business, and war, New York, 1950.

20. J. F. Nash, Non-cooperative games, Ann. of Math. vol. 54 (1951) pp. 286-295.

21. R. Otter and J. J. Dunne, Games with equilibrium points, Proc. Nat. Acad. Sci. U.S.A. vol. 39 (1953) pp. 310-314.

22. F. P. Ramsey, Truth and probability (1926), Further considerations (1928), Foundations of Mathematics and Other Logical Essays, London and New York, 1931.

23. M. Richardson, On weakly ordered systems, Bull. Amer. Math. Soc. vol. 52 (1946) pp. 113-116; Extension theorems for solutions of irreflexive relations, Proc. Nat. Acad. Sci. U.S.A. vol. 39 (1953) pp. 649-655; Solutions of irreflexive relations, Ann. of Math. vol. 58 (1953) pp. 573-590; Relativization and extension of solutions of irreflexive relations, Pacific J. Math. vol. 5 (1955) pp. 551-584.

24. L. J. Savage, The foundations of statistics, New York, 1954.

25. L. S. Shapley, Stochastic games, Proc. Nat. Acad. Sci. U.S.A. vol. 39 (1953) pp. $1095-1100$.

26. J. Ville, Sur la theorie generale des jeux ou intervient l'habilete des joueurs, Traité du Calcul des Probabilités et de ses Applications (by E. Borel and collaborators) vol. IV, no. 2, Paris, 1938, pp. 105-113.

27. A. Wald, Über die eindeutige positive Lösbarkeit der neuen Produktionsgleichungen, Ergeb. eines Math. Koll. Vienna (ed. by Karl Menger) vol. 6 (1935) pp. 1220; Über die Produktionsgleichungen der ökonomischen Wertlehre, ibid. vol. 7 (1936) pp. 1-6; Über einige Gleichungssysteme der mathematischen Ökonomie, Z. Nationalökonomie vol. 7 (1936) pp. 637-670 [English translation in Econometrica vol. 19 (1951) pp. 368-403].

28. - Generalization of a theorem by v. Neumann concerning zero sum two person games, Ann. of Math. vol. 46 (1945) pp. 281-286; Statistical decision functions which minimize the maximum risk, ibid. pp. 265-280; Statistical decision functions, New York, 1950.

29. P. Wolfe, The strict determinateness of certain infinite games, Pacific J. Math. vol. 5 (1955) pp. 841-847.

30. E. Zermelo, Über eine Anwendung der Mengenlehre auf die Theorie des Schachspiels, Proceedings of the Fifth International Congress of Mathematicians, Cambridge, 1912, vol. 2, p. 501-504.

\section{Bryn Mawr College and} PRINCETON UNIVERSITY 